\title{
OPEN The value of ventricular gradient for predicting pulmonary hypertension and mortality in hemodialysis patients
}

\author{
A. Jaroszyński ${ }^{1,2 \varpi}$, T. T. Schlege ${ }^{3,4}$, T. Zaborowski ${ }^{5}$, T. Zapolski $^{6}$, W. Załuska ${ }^{7}$, \\ A. Janion-Sadowska ${ }^{1}$, D. Kozieł ${ }^{8}$, S. Głuszek ${ }^{1} \&$ W. Dąbrowski ${ }^{9}$
}

Pulmonary hypertension (PHT) is associated with increased mortality in hemodialysis (HD) patients. The ventricular gradient optimized for right ventricular pressure overload (VG-RVPO) is sensitive to early changes in right ventricular overload. The study aimed to assess the ability of the VG-RVPO to detect PHT and predict all-cause and cardiac mortality in HD patients. 265 selected HD patients were enrolled. Clinical, biochemical, electrocardiographic, and echocardiographic parameters were evaluated. Patients were divided into normal and abnormal VG-RVPO groups, and were followed-up for 3 years. Abnormal VG-RVPO patients were more likely to be at high or intermediate risk for PHT, were older, had longer HD vintage, higher prevalence of myocardial infarction, higher parathormone levels, shorter pulmonary flow acceleration time, lower left ventricular ejection fraction, higher values of left atrial volume index, left ventricular mass index, and peak tricuspid regurgitant velocity. Both all-cause and CV mortality were higher in abnormal VG-RVPO group. In multivariate Cox analysis, VG-RVPO remained an independent and strong predictor of all-cause and CV mortality. In HD patients, abnormal VG-RVPO not only predicts PHT, but also all-cause and CV mortality.

Pulmonary hypertension (PHT) is prevalent in hemodialysis (HD) patients, and is associated with increased morbidity and mortality. The high incidence of PHT in HD patients relates not only to left-sided heart disease, but also to HD-specific factors. The deterioration of kidney function per se may be a trigger for the development of PHT, and the uremic milieu leads to pulmonary vasoconstriction and decreased compliance of the pulmonary vasculature $^{1-7}$. Mechanisms responsible for PHT in HD patients have not been completely understood. However, abnormal endothelium-dependent vasodilatation, vascular calcification, thromboembolic disease, hypervolemia, increased pulmonary vascular flow due to the presence of arterio-venous fistulas, anemia and sleep-disordered breathing play a role ${ }^{7-10}$. The classical symptoms of PHT occur relatively late and may be confounded by overhydration in HD patients, leading to the delayed diagnosis and the worsening of prognosis ${ }^{11}$. To enable earlier detection of PHT, regular screening by use of noninvasive tools is advisable. However, the diagnostic accuracy of standard electrocardiographic (ECG) parameters is low in detecting $\mathrm{PHT}^{10,12-16}$.

Recently, there has been renewed interest in the ventricular gradient (VG) to quantify ventricular action potential duration (APD) heterogeneity. The spatial VG is the vectorial sum of spatial QRS and spatial T integral vectors. VG runs from the area of the longest mean APD toward the area of myocardium having the shortest APD. Owing to this fact VG is considered a potential marker of increased CV risk, including sudden death. Changes in magnitude and/or orientation of the VG reflect APD inhomogeneities related to electrical RV remodelling as a result of an increased RV pressure load. Previously published studies have demonstrated that spatial VG, and especially ventricular gradient optimized for right ventricular pressure overload (VG-RVPO) was a useful CV predictor in some clinical settings ${ }^{16-27}$. VG-RVPO is the VG vector sum in a specific spatial direction providing an optimized projection for detection of RV pressure overload (VG magnitude at elevation $27^{\circ}$ and azimuth

${ }^{1}$ Collegium Medicum, Jan Kochanowski University in Kielce, Al. IX Wieków Kielc 19A, 25-317, Kielce, Poland. ${ }^{2}$ Department of Nephrology, Wojewódzki Szpital Zespolony in Kielce, Kielce, Poland. ${ }^{3}$ Department of Molecular Medicine and Surgery, Karolinska Institutet, Stockholm, Sweden. ${ }^{4}$ Nicollier-Schlegel Sàrl, Trélex, Switzerland. ${ }^{5}$ Department of Family Medicine, Medical University of Lublin, Lublin, Poland. ${ }^{6}$ Department of Cardiology, Medical University of Lublin, Lublin, Poland. 'Department of Nephrology, Medical University of Lublin, Lublin, Poland. ${ }^{8}$ Institute of Public Health, Jan Kochanowski University in Kielce, Kielce, Poland. ${ }^{9}$ Department of Anesthesiology and Intensive Care, Medical University of Lublin, Lublin, Poland. ${ }^{\circledR}$ email: jaroszynskiaj@interia.pl 
$\left.155^{\circ}\right)^{16,23,25-27}$. Several studies have revealed that VG-RVPO can accurately detect increased pulmonary pressures, is sensitive to early changes in RV overload, and has higher diagnostic accuracy for elevated RV pressure than other known ECG-derived parameters ${ }^{14,16,19,20,25,27,28}$. However, the prognostic value of VG-RVPO has not been evaluated in HD patients.

The purpose of the study was to assess the ability of VG-RVPO to detect PHT and predict all-cause as well as cardiac mortality in HD patients.

\section{Material and methods}

Participant recruitment and selection. This prospective study was conducted in adult HD patients treated at two HD units in Lublin (Poland). Exclusion criteria applied in the present study were as follows: treatment $<3$ months (to include exclusively ESRD patients); advanced neoplastic disease; acute coronary syndrome or stroke over the prior 3 months (to exclude the influence of acute ischemia on VG-RVPO values); electronic pacing; or symptoms of acute infection or hemorrhage at baseline (to reduce the possible influence of transient factors on VG-RVPO values).

Owing to the fact that it was impossible to estimate population size meeting the criteria described above, a prospective sample size calculation was not performed. All HD patients in Lublin were invited and those who decided to participate were included. Written informed consent was obtained from all participants. The study was approved by the Ethical Committee of Medical University of Lublin (KE-0254/125/2011) and conducted in accordance with the Declaration of Helsinki.

Control group. ECG was performed in 50 gender- (26 F and $24 \mathrm{M})$ and age-matched (69.3 \pm 6.1 years) healthy volunteers who had normal laboratory tests and displayed no abnormalities detected by physical examination and chest $\mathrm{x}$-ray.

Biochemical parameters. Routinely determined biochemical parameters were measured at the beginning of the follow-up (the day after the HD session) at the same time as both echocardiography and electrocardiography. The following parameters were evaluated: sodium, potassium, calcium, phosphorus, creatinine, urea, hemoglobin, parathormone $(\mathrm{PTH})$, total protein, albumin, C-reactive protein (CRP), total cholesterol, HDL cholesterol, LDL cholesterol, triglycerides (TG) and troponin T. Blood was obtained in the morning after at least $8 \mathrm{~h}$ fasting.

Electrocardiographic parameters. Surface 12-lead resting ECG was recorded in each patient and in controls using a MEDEA device (Kardio PCM-u, Poland). ECGs were recorded in an electrically shielded and noise-proof room with subjects lying in the horizontal position after at least 5 min rest. All recordings were obtained during the short, inter-dialysis interval. The $10 \mathrm{~s}$ recordings were automatically averaged to a single beat, and transformed into three orthogonal leads X, Y and Z using the inverse Dower method. The areas under the curves of the QRST complexes in X-, Y-, and Z leads were calculated automatically by integrating voltages over the entire QRST complex from the MEDEA software (licence number 245/2018, http://medea.pl/kompu terowy-aparat-ekg/). VG-RVPO was calculated using trigonometric equation: VG-RVPO $=-0.807526151172$ * $\mathrm{VGx}+0.404508497187 * \mathrm{VGy}+0.376555628452 * \mathrm{VGz}$ where VGx, VGy and VGz are the areas under the X-, Y-, and Z-components of the VCG, respectively (Fig. 1).

Echocardiographic examination. Transthoracic echocardiography was performed in the morning after dialysis sessions s $^{293}$ using a 2.5-3.5 MHz probe and Sonos 5500 and 7500 units (Philips, Andover, MA, USA) according to American Society of Echocardiography recommendations ${ }^{30-32}$. The experienced cardiologist who performed echocardiography was blinded to all other clinical data. According to the guidelines of echocardiographic assessment of PHT, peak tricuspid valve velocity (TRV) was measured as the key parameter in determining the probability of PHT ${ }^{13,32,33}$. TRV was measured by continuous wave Doppler. The optimal window at parasternal short axis (PSAX), A4C view, subcostal view or a modified view between the PSAX and A4C was obtained. Then measurement from that complete tricuspid regurgitation envelope that had the highest velocity was performed. An average of three beats in sinus rhythm and five beats in atrial fibrillation was calculated. A TRV $<2.8 \mathrm{~m} / \mathrm{s}$ was considered normal. In addition to TRV measurements, echocardiographic parameters suggestive of PHT originating from the heart ventricles, pulmonary artery, and inferior vena cava and right atrium were assessed. These included, for example, the pulmonary flow acceleration time (ACT). Patients were then divided into low, intermediate, and high risk PHT groups based on their TRV values and the presence or absence of echocardiographic parameters mentioned above suggesting $\mathrm{PHT}^{13,32,33}$.

In addition to the echocardiographic parameters necessary to assess the probability of PHT, other echocardiographic parameters known to be prognostic factors in HD patients, such as left ventricular mass index (LVMI), left ventricular ejection fraction (LVEF), left atrial volume index (LAVI), and ratio between early mitral inflow velocity and mitral annular early diastolic velocity (E/e`), were also measured ${ }^{30,31}$.

Follow-up data. Patients were followed for 36 months or until the date of death or renal transplant starting from the day of baseline assessment. The end points of the current study were defined as all-cause and CV mortality. The definition of CV death was in line with that presented in Standardized Definitions for End Point Events in Cardiovascular Trials ${ }^{34}$. Events were determined by two independent physicians blinded to other results. If opinions were divergent, the event was verified by the third physician, an expert in cardiology. 


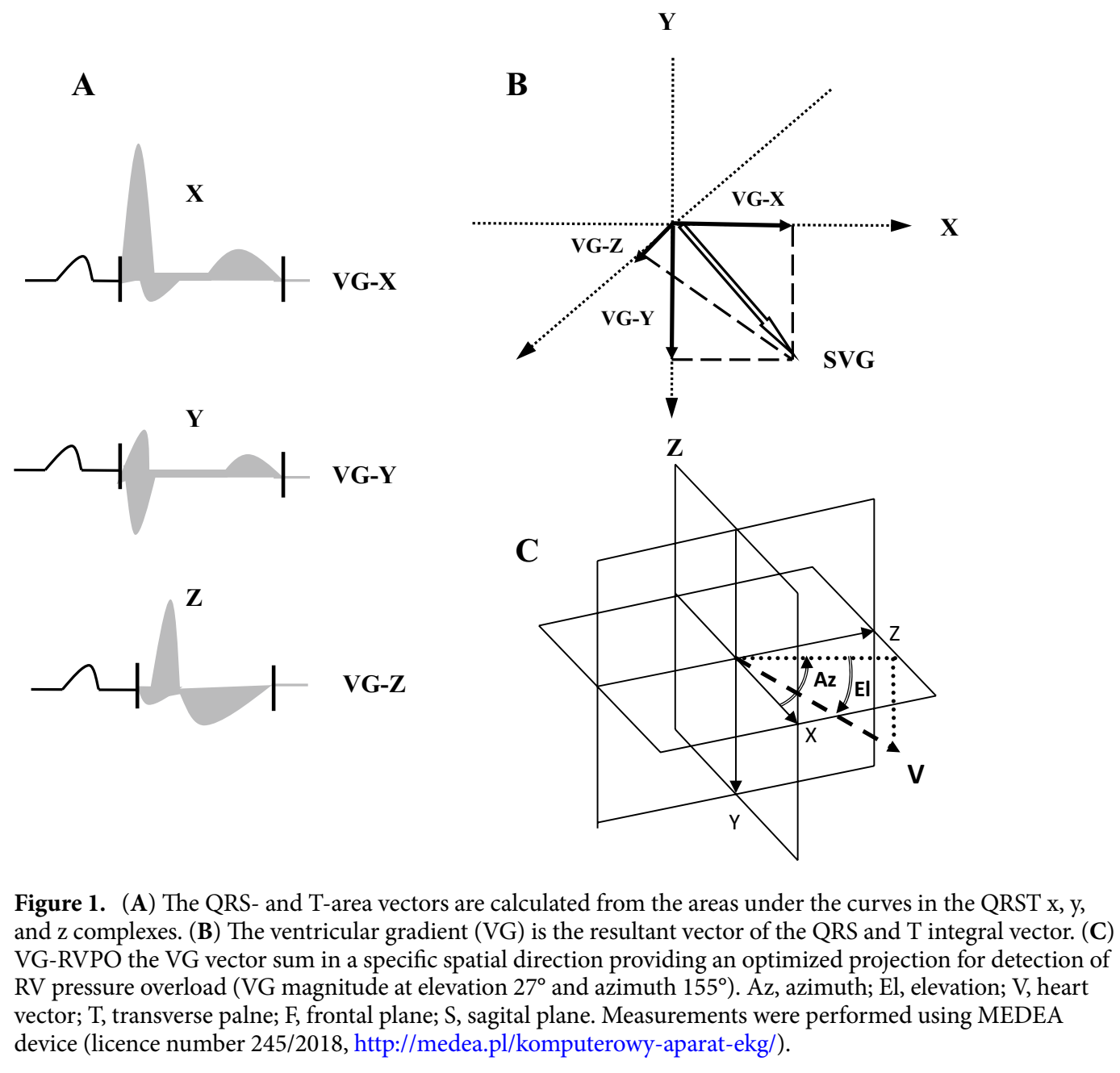

Statistical analysis. Statistical analysis was performed by using Statistica Version 10 as described in detail previously ${ }^{35}$. Initially the data were assessed for distribution by using Kolmogorov-Smirnov test. When normally distributed, continuous variables were expressed as mean $\pm \mathrm{SD}$, and as median and range when non-normally distributed. Categorical data were expressed as frequencies and percentages. For analysis, patients were divided into two groups depending on the VG-RVPO values. The VG-RVPO value $\geq-13 \mathrm{mV}^{\star} \mathrm{ms}$ was considered abnormal and $<-13 \mathrm{mV}^{\star} \mathrm{ms}$ was considered normal. The cut-off value was based on previous research results ${ }^{23,24,27}$. Student's t-test for independent variables was used in the case of normally distributed parameters. The Mann -Whitney U-test was used when results from at least one of the groups were not normally distributed. Linear regression analysis was performed using the Pearson or Spearman test, as appropriate. Multiple stepwise regression analysis was performed to include within the model only those measures that significantly differed ( $p$ value $<0.05$ ) between normal and abnormal VG-RVPO groups. The Kaplan-Meier method was used to assess the value of VG-RVPO for predicting all-cause and cardiac mortality in HD patients. Differences between patient groups were assessed using the log-rank test. A Cox proportional hazard regression analysis was also performed wherein only those variables with $p$ value $<0.15$ in the univariate analysis were considered for inclusion in the multivariate Cox model. Statistical significance was defined as $p<0.05$.

\section{Results}

Patients' clinical characteristics. Of the 303 available HD patients, 38 were excluded due to: treatment $<3$ months $(n=8)$, advanced neoplastic disease $(n=7)$, acute coronary syndrome or stroke over the period of 3 months $(n=4)$, pacing $(n=11)$, and acute infections or hemorrhages $(n=8)$.

The remaining $265 \mathrm{HD}$ patients (143 females and 122 males), aged $32-87$ years (mean $70.2 \pm 8.04$ ) were included. The mean HD vintage was $45.93 \pm 21.11$ (from 3 to 156) months. The causes of renal failure were as follows: diabetes $(n=103)$, chronic glomerulonephritis $(n=73)$, hypertensive nephropathy $(n=22)$, polycystic kidney disease $(n=10)$, obstructive nephropathy $(n=11)$, chronic pyelonephritis $(n=7)$, and unknown/unsure $(\mathrm{n}=39)$. Clinical baseline characteristics of the studied patients are depicted in Table 1.

Out of 265 patients enrolled in the study, $71.3 \%$ were treated with angiotensin-converting enzyme inhibitors (ACEI) or angiotensin receptor blockers (ARB), 83.4\% received beta-blockers, and $53.9 \%$ statins. Hypertension 


\begin{tabular}{|c|c|c|c|c|}
\hline Parameter & All patients $n=265$ & VG-RVPO $\geq-13 \mathrm{mV}^{\star} \mathrm{ms} \mathrm{n}=81$ & VG-RVPO $<-13 \mathrm{mV}^{*} \mathrm{~ms} \mathrm{n}=184$ & $p$ \\
\hline Age (years) & $70.2 \pm 8.34$ & $72.4 \pm 8.18$ & $69.3 \pm 8.21$ & $<0.001$ \\
\hline $\operatorname{Sex}(F / M-n)$ & 1.18 & 1.17 & 1.18 & 0.763 \\
\hline HD vintage (months) & $45.9 \pm 21.11$ & $57.1 \pm 18.65$ & $41.8 \pm 19.31$ & $<0.001$ \\
\hline MI (\%) & 21.9 & 28.4 & 19.0 & 0.001 \\
\hline Diabetes mellitus (\%) & 51.9 & 50.6 & 54.3 & 0.234 \\
\hline Hypertension (\%) & 58.9 & 58.0 & 59.2 & 0.653 \\
\hline Smoking & 10.2 & 8.6 & 10.8 & 0.196 \\
\hline Beta-blockers (\%) & 83.4 & 86.4 & 82.1 & 0.227 \\
\hline ACEI/ARB (\%) & 71.3 & 74.0 & 70.1 & 0.314 \\
\hline Statins (\%) & 53.9 & 55.5 & 53.3 & 0.299 \\
\hline $\mathrm{E} / \mathrm{e}^{\prime}(\mathrm{n})$ & $14.51 \pm 5.11$ & $15.19 \pm 4.99$ & $13.96 \pm 5.11$ & 0.195 \\
\hline $\operatorname{LVMI}\left(\mathrm{g} / \mathrm{m}^{2}\right)$ & $145.2 \pm 41.12$ & $156.1 \pm 36.09$ & $141.4 \pm 38.76$ & $<0.001$ \\
\hline LVEF (\%) & $55.13 \pm 5.11$ & $51.56 \pm 5.11$ & $56.64 \pm 5.22$ & $<0.001$ \\
\hline $\mathrm{TRV}(\mathrm{m} / \mathrm{s})$ & $2.49 \pm 0.67$ & $3.69 \pm 0.70$ & $1.92 \pm 0.65$ & $<0.001$ \\
\hline ACT (ms) & $101.6 \pm 27$ & $74.5 \pm 24$ & $113 \pm 25$ & $<0.001$ \\
\hline LAVI $\left(\mathrm{ml} / \mathrm{m}^{2}\right)$ & $36.75 \pm 8.04$ & $39.03 \pm 7.64$ & $35.86 \pm 7.72$ & 0.001 \\
\hline High PHT risk (\%) & 20.0 & 54.3 & 4.9 & $<0.001$ \\
\hline Intermediate PHT risk (\%) & 35.5 & 45.7 & 31.0 & $<0.001$ \\
\hline Low PHT risk (\%) & 44.5 & 0.0 & 64.1 & $<0.001$ \\
\hline Hemoglobin (g/dL) & $11.03 \pm 1.08$ & $10.91 \pm 1.01$ & $11.10 \pm 1.22$ & 0.569 \\
\hline Total cholesterol $(\mathrm{mg} / \mathrm{dL})$ & $187.1 \pm 36.71$ & $188.1 \pm 36.22$ & $186.9 \pm 35.14$ & 0.681 \\
\hline LDL cholesterol (mg/dL) & $117.3 \pm 29.65$ & $116.1 \pm 29.15$ & $118.3 \pm 30.01$ & 0.524 \\
\hline HDL cholesterol (mg/dL) & $44.02 \pm 17.35$ & $45.1 \pm 16.18$ & $44.00 \pm 16.55$ & 0.723 \\
\hline Triglycerides (mg/dL) & $173.4 \pm 59.13$ & $171.0 \pm 56.86$ & $174.2 \pm 56.5$ & 0.435 \\
\hline $\mathrm{PTH}$, range $(\mathrm{pg} / \mathrm{mL})$ & $415(0.0-1278)$ & $487(0.0-1278)$ & $402(0.0-1006)$ & $<0.001$ \\
\hline Albumin (g/dL) & $3.69 \pm 0.31$ & $3.55 \pm 0.32$ & $3.73 \pm 0.30$ & 0.116 \\
\hline $\mathrm{CRP}$, range $(\mathrm{mg} / \mathrm{dL})$ & $8.15(0.31-95.1)$ & $9.74(0.31-61.2)$ & $7.59(0.91-95.1)$ & 0.207 \\
\hline Troponin T, range $(\mu \mathrm{g} / \mathrm{L})$ & $0.043(0.00-0.702)$ & $0.061(0.035-0.702)$ & $0.040(0.00-0.597)$ & 0.132 \\
\hline Sodium (mmol/L) & $137.5 \pm 2.63$ & $137.9 \pm 2.56$ & $137.4 \pm 2.79$ & 0.871 \\
\hline Potassium (mmol/L) & $5.43 \pm 0.72$ & $5.58 \pm 0.74$ & $5.37 \pm 0.63$ & 0.342 \\
\hline Calcium $(\mathrm{mmol} / \mathrm{L})$ & $2.46 \pm 0.22$ & $2.48 \pm 0.24$ & $2.45 \pm 0.25$ & 0.389 \\
\hline Phosphate (mmol/L) & $2.27 \pm 0.27$ & $2.30 \pm 0.25$ & $2.25 \pm 0.24$ & 0.307 \\
\hline
\end{tabular}

Table 1. Baseline characteristics of patients. MI, myocardial infarction; ACEI, inhibitors-angiotensin converting enzyme inhibitors; ARB, angiotensin 2 receptor blockers; E/e', ratio between early mitral inflow velocity and mitral annular early diastolic velocity; LVMI, left ventricular mass index; LVEF, left ventricle ejection fraction; TRV, peak tricuspid regurgitant velocity; AcT, pulmonary flow acceleration time; LAVI, left atrial volume index; PHT, pulmonary hypertension; PTH, parathormone; CRP, C-reactive protein.

was present in $58.9 \%$ of patients, history of myocardial infarction in $21.9 \%$, and left ventricular hypertrophy $(\mathrm{LVH})$ in $65.3 \%$.

VG-RVPO comparison between patients and controls. VG-RVPO was less negative in patients compared with controls $(-20.72 \pm 16.35 \mathrm{mV} \cdot \mathrm{ms}$ vs. $-31.15 \pm 8.62 \mathrm{mV} \cdot \mathrm{ms}, p<0.001)$. No difference was found between VG-RVPO in HD females and males ( $-20.98 \pm 14.33$ vs. $-20.57 \pm 15.13, p=0.737)$.

Differences between patient groups. Patients in the abnormal VG-RVPO group were more likely to be at high or intermediate risk for PHT ( $p<0.001$ in both cases), and none of the patients from the abnormal VGRVPO group was at low risk for PHT $(p<0.001)$. Only $4.9 \%$ of the patients from the normal VG-RVPO group were at high risk for PHT. Patients in the abnormal VG-RVPO group were older $(p<0.001)$, had longer HD vintage $(p<0.001)$, and higher prevalence of MI $(p=0.001)$. With regard to biochemical indices, the abnormal VG-RVPO group also had higher PTH levels $(p<0.001)$. In relation to echocardiographic parameters, patients in the abnormal VG-RVPO group had shorter AcT (pulmonary flow acceleration time) $(p<0.001)$, lower LVEF values $(p<0.001)$, higher LAVI $(p=0.001)$, higher LVMI $(p<0.001)$, and higher TRV values $(p<0.001)$.

On the basis of echocardiographic criteria patients were divided into 3 groups according to PHT risk (low, intermediate and high). Differences in VG-RVPO values were found between the high and low risk groups $(-6.9 \pm 13.9$ vs. $-25.9 \pm 16.4, p<0.001)$, and the high and intermediate risk groups $(-6.9 \pm 13.9$ vs. $-18.2 \pm 15.1$, $p<0.001)$. The difference between the intermediate and low risks groups was also significant, however less pronounced $(-18.2 \pm 15.1$ vs. $-25.9 \pm 16.4, p=0.018)$. Likewise, differences in AcT values were found between the low and high risk groups $(109.1 \pm 21$ vs. $82.02 \pm 22.73, p<0.001)$, and between the low and intermediate groups 


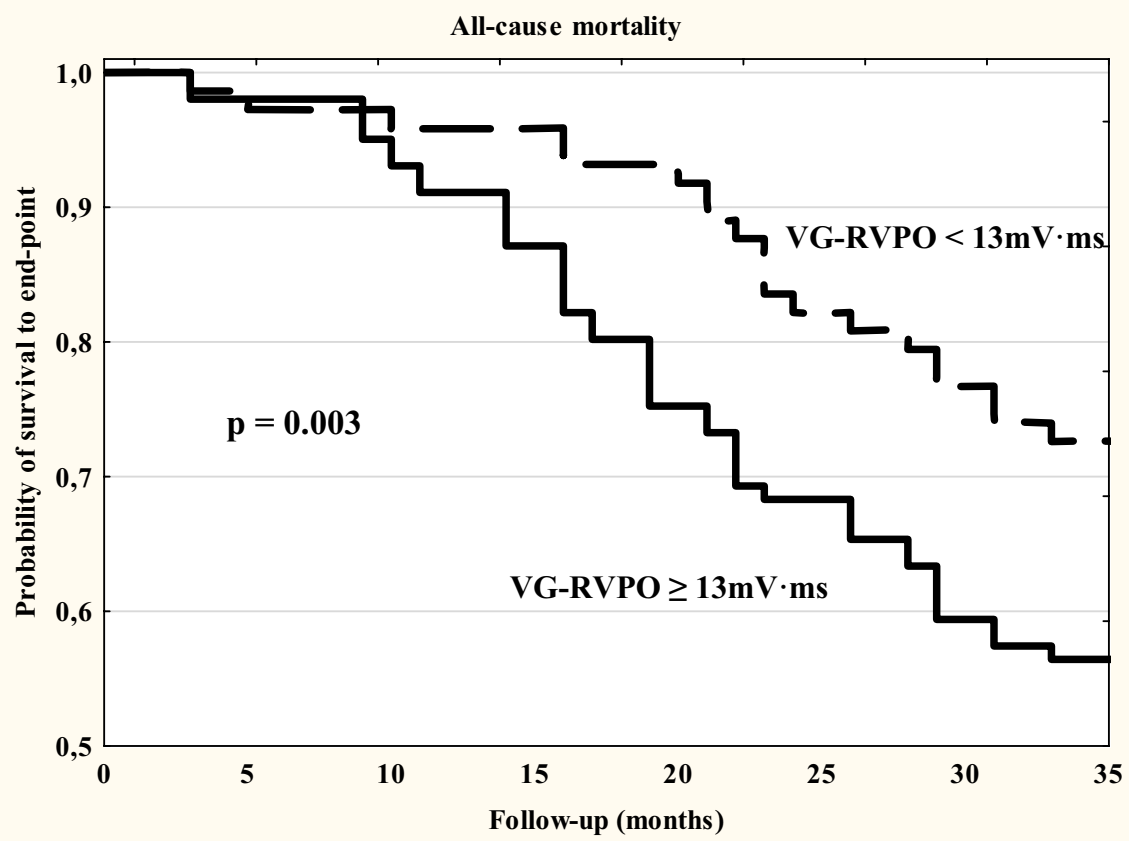

Figure 2. Survival plots analysis for all-cause mortality in HD patients stratified by normal and abnormal VG-RVPO value.

(109.1 \pm 21 vs. $96.3 \pm 20.1, p=0.017$ ). The difference in ACT between intermediate and high risk groups did not reach significance $(96.3 \pm 20.1$ vs. $109.1 \pm 21, p=0.119)$. Moreover, VG-RVPO correlated negatively with TRV $(\mathrm{r}=-0.682), p<0.001)$ and positively with ACT $(\mathrm{r}=0.611, p<0.001)$.

Survival and multivariate analysis. During the mean follow-up period of $26.9 \pm 4.8$ months, 99 all-cause deaths were noted $(37.5 \%)$. The mortality rate was $12.5 \%$ per year. Twenty three patients were transplanted. In the abnormal VG-RVPO group the incidence of all-cause death was $61.7 \%$ and was higher than that of the normal VG-RVPO group $(26.6 \%, p<0.001)$.

$\mathrm{CV}$ death contributed to $49.5 \%$ of all deaths. The proportion of CV death in the abnormal VG-RVPO group (72.1\%) was higher than that in the normal VG-RVPO group $(31.3 \%, p<0.001)$. Out of 21 transplanted patients only 2 came from the abnormal VG-RVPO group.

By Kaplan-Meier analysis, the cumulative incidence of both all-cause and CV mortality was higher in the abnormal VG-RVPO group then the normal VG-RVPO group; (-log-rank, $p=0.003$ and $p<0.001$, respectively (Figs. 2 and 3).

Multivariate Cox proportional hazard regression analyses were performed using a model consisting of univariate predictors of cardiac mortality to control for possible confounders. The results of both univariate and multivariate Cox proportional hazard regression analyses are presented in Tables 2 and 3. A multivariate analysis revealed that age [hazard ratio (HR) $1.76, p<0.001$ ], TRV (HR 1.37, $p=0.018$ ], LVMI (HR 1.79, $p=004$ ), and VG-RVPO (HR 2.01, $p=0.002$ ) were independent predictors of all-cause mortality. In the case of CV mortality, multivariate Cox proportional analysis indicated age (HR 2.19, $p<0.001$ ), HD vintage (HR 1.76, $p=0.006$ ), TRV (HR 1.71, $p=0.019$ ), LVMI (HR 1.72, $p=0.007)$, LAVI (HR 1.77, $p=0.011)$ and VG-RVPO (HR 2.40, $p<0.001)$ as independent predictors of CV mortality.

\section{Discussion}

The two major findings of the present study were that: (1) VG-RVPO corresponds with PHT and correlates with echocardiographic parameters related to PHT; and (2) VG-RVPO is an independent predictor of both all-cause and CV mortality in HD patients.

In the present study VG-RVPO values were higher in HD patients compared to controls, and $55.5 \%$ of patients were classified into intermediate and high risk groups for PHT based on echocardiographic parameters. This suggests that $\mathrm{PHT}$ is prevalent in HD patients, a finding in agreement with previous studies demonstrating that the prevalence of PHT is around 18.8-68.8\%, depending on the criteria adopted for PHT diagnosis, selection factors and co-morbidities ${ }^{2,5,8,10,36,37}$. Right heart catheterization was not included in the study protocol because of medical-ethical reasons. We were therefore unable to estimate the prevalence of PHT but instead only the risk of PHT. Given that echocardiography is only a screening tool for $\mathrm{PHT}^{32,33}$, our results should therefore be further corroborated in studies using RV catheterization.

To our knowledge this is the first study to demonstrate the usefulness of VG-RVPO in PHT prediction in HD patients. In the present study VG-RVPOcorresponded with PHT and correlated with echocardiographic parameters related to PHT, suggesting its usefulness in PHT screening and/or monitoring. Given that clinical 


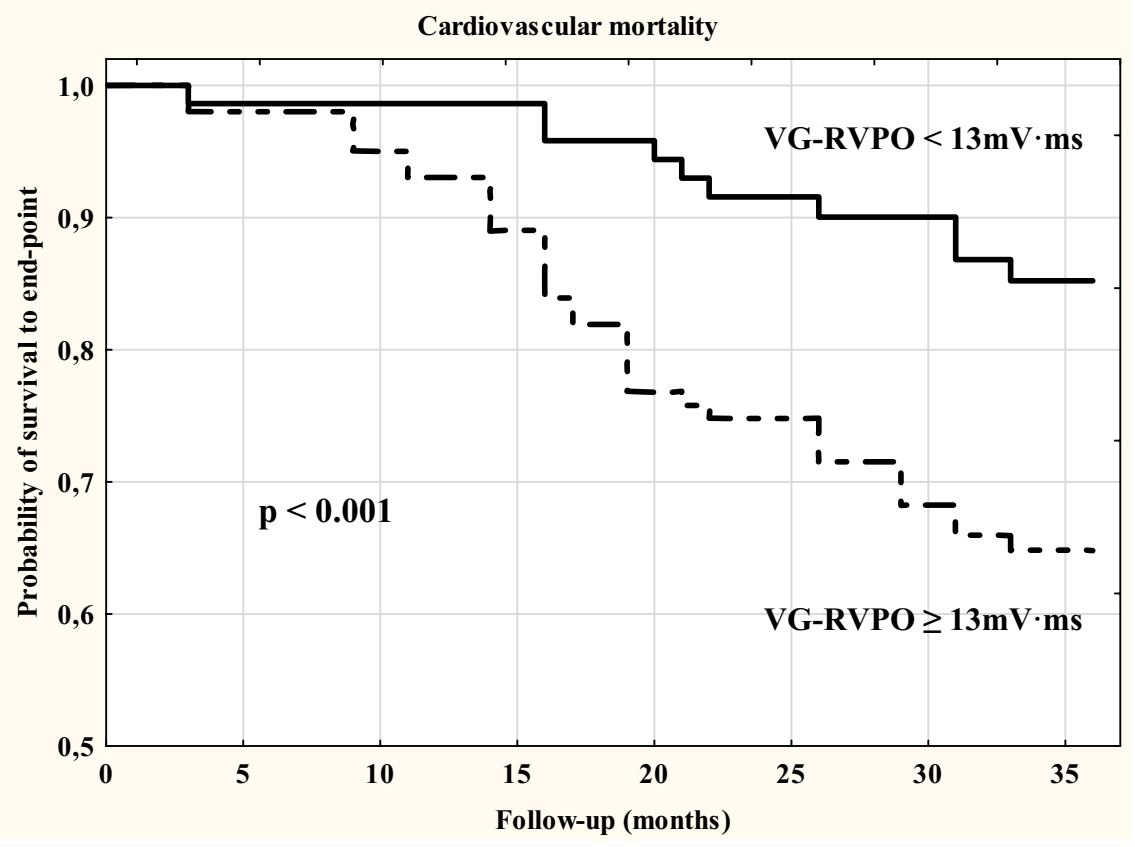

Figure 3. Survival plots analysis for cardiovascular mortality in HD patients stratified by normal and abnormal VG-RVPO value.

\begin{tabular}{|l|l|l|l|l|}
\hline Parameter & Univariate HR $(\mathbf{9 5} \% \mathbf{C I})$ & $\boldsymbol{P}$ & Multivariate HR $(\mathbf{9 5 \%}$ CI $)$ & $\boldsymbol{P}$ \\
\hline Age & $1.85(1.36-2.51)$ & $P<0.001$ & $1.76(1.19-2.63)$ & $<0.001$ \\
\hline HD vintage & $1.49(0.84-1.99)$ & 0.008 & $1.21(0.58-2.13)$ & 0.212 \\
\hline History of MI & $1.69(0.85-2.51)$ & 0.063 & $1.37(0.64-2.273)$ & 0.102 \\
\hline TRV & $1.42(0.91-2.11)$ & 0.002 & $1.37(0.71-2.54)$ & 0.018 \\
\hline AcT & $0.85(0.28-1.88)$ & 0.031 & $0.91(0.41-2.49)$ & 0.167 \\
\hline LVMI & $2.26(1.77-2.65)$ & $<0.001$ & $0.90(0.52-1.73)$ & 0.004 \\
\hline EF & $0.82(0.49-1.67)$ & 0.021 & $1.37(0.95-3.82)$ & 0.118 \\
\hline LAVI & $1.87(1.17-2.65)$ & 0.016 & & 0.266 \\
\hline PTH & $2.31(1.18-3.97)$ & 0.174 & $2.01(1.64-2.70)$ & 0.002 \\
\hline VG-RVPO & $2.29(1.83-2.73)$ & $<0.001$ & \\
\hline
\end{tabular}

Table 2. Uni- and multivariate predictors of all-cause mortality. HR, hazard ratio; CI, confidence interval; HD, hemodialysis; MI, myocardial infarction; TRV, peak tricuspid regurgitant velocity; AcT, pulmonary flow acceleration time; LVMI, left ventricular mass index; LVEF--left ventricle ejection fraction; LAVI-left atrial volume index; PTH, parathormone; VGx, ventricular gradient projected on the $\mathrm{x}$-axis. In the multivariate analyses, parameters with a $p \leq 0.15$ were entered.

symptoms of PHT are nonspecific and often masked by the presence of overhydration, pulmonary and heart diseases, the diagnosis of PHT is often delayed in HD patients, worsening prognosis ${ }^{11}$. While RV catheterization is the "gold standard" of PHT diagnosis, simple, inexpensive, noninvasive diagnostic tests are needed to allow earlier detection of PHT. However, conventional surface ECG lacks diagnostic accuracy. PHT is characterized by increased RV pressure overload, and hence, increased RV wall tension which only over time can cause RV hypertrophy ${ }^{38}$. Thus anatomical remodeling of the heart is not needed for development of PHT, and PHT can also occur without RV hypertrophy. Myocardial action potentials change when RV pressure changes, due to mechanoelectrical feedback. This results in VG-RVPO changes reflecting RV pressure load. Given that standard ECG criteria rely on depolarization characteristics of hypertrophic RV, that may not yet have developed, unless and until RV hypertrophy occurs, the value of standard ECG parameters in early detection of increased pulmonary pressure is limited ${ }^{12,15,16,20,28}$.

The usefulness of VG-RVPO in assessing PHT in HD patients as determined in our study is in line with recently published papers, which have demonstrated that abnormal VG and especially VG-RVPO is strongly associated with PHT both in experimental ${ }^{20,28}$ and clinical studies ${ }^{16,23-28,38,40}$. The clinical usefulness of VG-RVPO in PHT detection was confirmed in scleroderma ${ }^{23,24}$, acute pulmonary embolism ${ }^{27}$, and patients with suspected $\mathrm{PHT}^{25}$. Additionally, VG-RVPO correlated strongly with pulmonary artery pressure ${ }^{25}$, and was suitable as a 


\begin{tabular}{|l|l|l|l|l|}
\hline Parameter & Univariate HR $(\mathbf{9 5} \% \mathbf{C I})$ & $\boldsymbol{p}$ & Multivariate HR $(\mathbf{9 5} \% \mathbf{C I})$ & $\boldsymbol{p}$ \\
\hline Age & $2.33(1.52-2.92)$ & $<0.001$ & $2.19(1.58-2.97)$ & $<0.001$ \\
\hline HD vintage & $1.68(0.94-2.15)$ & 0.001 & $1.76(0.74-2.56)$ & 0.006 \\
\hline History of MI & $1.54(0.71-2.26)$ & 0.008 & $1.35(0.59-2.47)$ & 0.101 \\
\hline TRV & $1.91(1.04-2.66)$ & 0.004 & $1.71(0.99-2.96)$ & 0.019 \\
\hline AcT & $0.81(0.33-1.94)$ & 0.146 & $0.93(0.31-2.14)$ & 0.215 \\
\hline LVMI & $1.95(1.17-2.30)$ & $<0.001$ & $1.71(0.94-2.62)$ & 0.007 \\
\hline EF & $0.86(0.41-1.82)$ & 0.002 & $0.84(0.31-2.46)$ & 0.099 \\
\hline LAVI & $2.14(1.15-3.64)$ & 0.018 & $1.77(1.19-2.94)$ & 0.011 \\
\hline PTH & $2.71(1.45-3.76)$ & 0.012 & $2.14(1.26-3.58)$ & 0.044 \\
\hline VG-RVPO & $2.70(2.11-3.17)$ & $<0.001$ & $2.40(1.71-2.99)$ & $<0.001$ \\
\hline
\end{tabular}

Table 3. Uni- and multivariate predictors of cardiovascular mortality. HR, hazard ratio; CI, confidence interval; HD, hemodialysis; MI, myocardial infarction; TRV, peak tricuspid regurgitant velocity; AcT, pulmonary flow acceleration time; LVMI, left ventricular mass index; LVEF-left ventricle ejection fraction; LAVI-left atrial volume index; PTH, parathormone. In the multivariate analyses, parameters with a $p \leq 0.15$ were entered.

monitoring tool reflecting pulmonary artery pressure changes in patients with $\mathrm{PHT}^{38}$. Moreover, in a group of patients with scleroderma VG-RVPO was superior to echocardiography derived parameters for predicting increased RV load as well as to detect disease progression in early phases of the disease ${ }^{24,26}$.

Our study suggests that VG-RVPO is an independent and strong predictor of both all-cause and CV mortality in HD patients. This finding is in line with previous observations that the VG is a risk predictor both in the general population ${ }^{38}$ and in post-infarction patients ${ }^{22}$. The spatial VG has also been associated with sudden cardiac death in the general population ${ }^{19}$. Abnormal VG-RVPO was also associated with increased mortality in patients with scleroderma, and the spatial VG vector projected on the $\mathrm{x}$-axis predicted all-cause mortality in PHT patients ${ }^{14}$.

The association between VG-RVPO and all-cause as well as CV mortality is probably due to the fact that VG-RVPO, like some other VG-based parameters, is a measure of APD heterogeneity, which is associated with increased susceptibility to ventricular arrhythmias. It is considered to be a marker of global electrical heterogeneity of the myocardium ${ }^{19,20,39}$. Any change in the myocardial electrical heterogeneity is associated with a change in VG-RVPO value. RV pressure load affects ventricular APD distribution resulting in a change in VGRVPO $^{16,19,20,40}$. Though VG-RVPO is the risk stratifier, especially suitable in PHT prediction, the knowledge about its physiologic correlates is limited and requires further study.

If our results are confirmed in future studies using right heart catheterization, VG-RVPO may become a useful tool for both the prediction and serial monitoring of PHT in HD patients.

\section{Limitation}

Our study has some limitations. First, we have evaluated exclusively echocardiographic parameters predictive of PHT but did not perform right heart catheterization, the gold standard in the diagnosis of PHT. We are aware of the limitations of echocardiography as a screening tool for PHT. However, echocardiography is accepted as the most useful screening tool for $\mathrm{PHT}^{32,33}$, and we recognize that without RV catheterizations, our results should only be construed as preliminary. Moreover, the fact that echocardiography was performed by only one cardiologist in our study may be a source of potential bias. Second, it is likely that serial rather than single VG-RVPO measurements may influence the results, making VG-RVPO either more or less useful in predicting PHT as well as clinical events in HD patients. Third, the inverse Dower transform utilized by our MEDEA device is not necessarily the scientifically most optimal transform for derivation of the X, Y and Z leads. Therefore, a more optimized transform and also a more fully automated software method would ideally be used in future studies to further optimize results and eliminate potential methodological bias ${ }^{41}$.

\section{Conclusions}

In HD patients, abnormal VG-RVPO not only predicts PHT, but also all-cause and CV mortality.

Received: 24 June 2021; Accepted: 9 December 2021

Published online: 10 January 2022

\section{References}

1. Navaneethan, S. et al. Prevalence, predictors, and outcomes of pulmonary hypertension in CKD. J. Am. Soc. Nephrol. 27, 877-886 (2016).

2. Sarnak, M. \& Roberts, K. Pulmonary hypertension in CKD: Some answers, yet more questions. J. Am. Soc. Nephrol. 27, 661-663 (2015).

3. O'Leary, J., Assad, T. \& Birdwell, K. Pulmonary hypertension in patients with chronic kidney disease: Invasive hemodynamic etiology and outcomes. Pulm. Circ. 7, 674-683 (2017).

4. Di Lullo, L. et al. Pulmonary hypertension and right heart failure in chronic kidney disease: New challenge for 21 st-century cardionephrologists. Cardiorenal. Med. 3, 96-103 (2013). 
5. Bolignano, D. et al. Pulmonary hypertension in CKD. Am. J. Kidney Dis. 61, 612-622 (2013).

6. Zhang, Y. et al. The prevalence of pulmonary hypertension among maintenance dialysis patients with ESRD and its associated factors: A retrospective study. Front Med. https://doi.org/10.3389/fmed.2020.570874 (2020).

7. Travers, A., Farber, H. \& Sarnak, M. Pulmonary hypertension in chronic kidney disease. Cardiol. Clin. 39, 427-434 (2021).

8. Kosmadakis, G., Aguilera, D., Carceles, O., Da Costa Correia, E. \& Boletis, I. Pulmonary hypertension in dialysis patients. Ren. Fail. 35, 514-520 (2013).

9. Agarwal, R. Prevalence, determinants and prognosis of pulmonary hypertension among hemodialysis patients. Nephrol. Dial. Transplant. 27, 3908-4391 (2012).

10. Zhang, Q., Wang, L., Zeng, H., Lv, Y. \& Huang, Y. Epidemiology and risk factors in CKD patients with pulmonary hypertension: A retrospective study. BMC Nephrol. 19, 70. https://doi.org/10.1186/s12882-018-0866-9 (2018).

11. Humbert, M., Coghlan, J. \& Khanna, D. Early detection and management of pulmonary arterial hypertension. Eur. Respir. Rev. 21, 306-312 (2012).

12. Kovacs, G. et al. Use of ECG and other simple non-invasive tools to assess pulmonary hypertension. PLoS ONE https://doi.org/ 10.1371/journal.pone.0168706 (2016).

13. Galiè, N. et al. ESC Scientific Document Group: 2015 ESC/ERS Guidelines for the diagnosis and treatment of pulmonary hypertension. Eur. Heart J. 37, 67-119 (2016).

14. Scherptong, R. et al. Diagnosis and mortality prediction in pulmonary hypertension: The value of the electrocardiogram-derived ventricular gradient. J. Electrocardiol. 45, 312-318 (2012).

15. McGoon, M. et al. Screening, early detection, and diagnosis of pulmonary arterial hypertension: ACCP evidence-based clinical practice guidelines. Chest 126, 14-34 (2004).

16. Henkens, I. et al. Improved ECG detection of presence and severity of right ventricular pressure load validated with cardiac magnetic resonance imaging. Am. J. Physiol. Heart Circ. Physiol. 294, 2150-2157 (2008).

17. Treskes, R. et al. Performance of ST and ventricular gradient difference vectors in electrocardiographic detection of acute myocardial ischemia. J. Electrocardiol. 48, 498-504 (2015).

18. Man, S. et al. Predictive power of T-wave alternans and of ventricular gradient hysteresis for the occurrence of ventricular arrhythmias in primary prevention cardioverter-defibrillator patients. J. Electrocardiol. 44, 453-459 (2011).

19. Waks, J. et al. Global electrical heterogeneity risk score for prediction of sudden cardiac death in the general population: The atherosclerosis risk in communities (ARIC) and cardiovascular health (CHS) studies. Circulation 133, 2222-2234 (2016).

20. Waks, J. \& Tereshchenko, L. Global electrical heterogeneity: A review of the spatial ventricular gradient. J. Electrocardiol. 49, $824-830$ (2016).

21. Pan, D., Liu, R., Ren, S., Li, C. \& Chang, G. Prediction of pulmonary arterial hypertension in chronic obstructive lung disease from three-dimensional vectorcardiographic parameters. Ann. Noninvasive. Electrocardiol. 21, 280-286 (2016).

22. Zabel, M. et al. Analysis of 12-lead T-wave morphology for risk stratification after myocardial infarction. Circulation 102, $1252-$ $1257(2000)$.

23. Couperus, L. et al. Electrocardiographic detection of pulmonary hypertension in patients with systemic sclerosis using the ventricular gradient. J. Electrocardiol. 49, 60-68 (2016).

24. Meijer, F. et al. ECG derived ventricular gradient exceeds echocardiography in the early detection of pulmonary hypertension in scleroderma patients. Int. J. Cardiol. 273, 203-206 (2018).

25. Kamphuis, V. et al. Electrocardiographic detection of right ventricular pressure overload in patients with suspected pulmonary hypertension. J. Electrocardiol. 47, 175-182 (2014).

26. Nielsen-Kudsk, J., Andersen, S. \& Andersen, A. Can computer assisted analysis of a standard 12-lead ECG be used for early diagnosis and monitoring of disease progression in pulmonary hypertension?. Int. J. Cardiol. 273, 221-222 (2018).

27. Meijer, F. et al. The prognostic value of ECG-derived ventricular gradient in early adverse events in acute pulmonary embolism patients. Thrombosis Update 2, 100033 (2021).

28. Henkens, I. et al. Early changes in rat hearts with developing pulmonary arterial hypertension can be detected with three-dimensional electrocardiography. Am. J. Physiol. Heart Circ. Physiol. 293, 1300-1307 (2007).

29. Stewart, G. et al. Electrocardiographic abnormalities and uremic cardiomyopathy. Kidney Int. 67, 217-226 (2005).

30. Hickson, L. et al. Echocardiography criteria for structural heart disease in patients with end-stage renal disease initiating hemodialysis. J. Am. Coll. Cardiol. 67, 1173-1182 (2016).

31. Lang, R. et al. Recommendations for cardiac chamber quantification by echocardiography in adults: An update from the American society of echocardiography and the European association of cardiovascular imaging. J. Am. Soc. Echocardiogr. 28, 1-39 (2015).

32. Douglas, P. et al. 2019 ACC/AHA/ASE key data elements and definitions for transthoracic echocardiography: A report of the american college of cardiology/American heart association task force on clinical data standards (writing committee to develop clinical data standards for transthoracic echocardiography) and the American society of echocardiography. Circ. Cardiovasc. Imaging. 12, e000027. https://doi.org/10.1161/HCI.0000000000000027 (2019).

33. Augustine, D. et al. Echocardiographic assessment of pulmonary hypertension: A guideline protocol from the British Society of Echocardiography. Echo. Res. Pract. 5(3), G11-G24. https://doi.org/10.1530/ERP-17-0071 (2018).

34. Hicks, K. et al. Cardiovascular and Stroke endpoint definitions for clinical trials. Circulation 2018(137), 961-972 (2017).

35. Jaroszyński, A. et al. The prognostic value of positive T-wave in lead aVR in hemodialysis patients. Clin. Exp. Nephrol. 19, 1157-1164 (2015).

36. Nishimura, M. et al. Idiopathic pre-capillary pulmonary hypertension in patients with end-stage kidney disease: Effect of endothelin receptor antagonists. Clin. Exp. Nephrol. 21, 1088-1096 (2017).

37. Li, Z. et al. Pulmonary hypertension: Epidemiology in different CKD stages and its association with cardiovascular morbidity. PLoS ONE 9, el14392 (2014).

38. de Jongh, M. et al. Electrocardiographic detection and monitoring of pulmonary hypertension. Comput. Cardiol. 42, 541-544 (2015).

39. Malik, M. Ventricular gradient and cardiac risk. Europace 13, 605-607 (2011).

40. Draisma, H., Schalij, M., van der Wall, E. \& Swenne, C. Elucidation of the spatial ventricular gradient and its link with dispersion of repolarization. Heart Rhythm 3, 1092-1109 (2006).

41. Cortez, D. \& Schlegel, T. When deriving the spatial QRS-T angle from the 12-lead electrocardiogram, which transform is more Frank: Regression or inverse Dower?. J. Electrocardiol 43, 302-309 (2010).

\section{Author contributions}

A.J. data collection and analysis, manuscript writing, T.S. data analysis T.Z. (Zapolski) data collection and figures preparation S.G. data analysis T.Z. (Zaborowski) data collection and analysis A.J-S. data analysis W.Z. data analysis W.D. data collection and anaylsis D.K. data collection All authors reviewed manuscript.

\section{Funding}

Uniwersytet Medyczny w Lublinie, DS 232. 


\section{Competing interests}

The authors declare no competing interests.

\section{Additional information}

Correspondence and requests for materials should be addressed to A.J.

Reprints and permissions information is available at www.nature.com/reprints.

Publisher's note Springer Nature remains neutral with regard to jurisdictional claims in published maps and institutional affiliations.

(c) (i) Open Access This article is licensed under a Creative Commons Attribution 4.0 International License, which permits use, sharing, adaptation, distribution and reproduction in any medium or format, as long as you give appropriate credit to the original author(s) and the source, provide a link to the Creative Commons licence, and indicate if changes were made. The images or other third party material in this article are included in the article's Creative Commons licence, unless indicated otherwise in a credit line to the material. If material is not included in the article's Creative Commons licence and your intended use is not permitted by statutory regulation or exceeds the permitted use, you will need to obtain permission directly from the copyright holder. To view a copy of this licence, visit http://creativecommons.org/licenses/by/4.0/.

(C) The Author(s) 2022 